The Role of Disk-Halo Interaction in Galaxy Evolution: Outflow vs Infall? Editor

EAS Publications Series, Vol. ?, 2018

\title{
DENSITY PDFS OF DIFFUSE GAS IN THE MILKY WAY
}

\author{
Elly M. Berkhuijsen ${ }^{1}$ and Andrew Fletcher ${ }^{2}$
}

\begin{abstract}
The probability distribution functions (PDFs) of the average densities of the diffuse ionized gas (DIG) and the diffuse atomic gas are close to lognormal, especially when lines of sight at $|b|<5^{\circ}$ and $|b| \geq 5^{\circ}$ are considered separately. Our results provide strong support for the existence of a lognormal density PDF in the diffuse ISM, consistent with a turbulent origin of density structure in the diffuse gas.
\end{abstract}

\section{Introduction}

Simulations of the interstellar medium (ISM) have shown that, if isothermal turbulence is shaping the structure of the medium, the density distribution becomes lognormal (Elmegreen \& Scalo, 2004, de Avillez \& Breitschwerdt, 2005; Wada \& Norman, 2007). The latter authors found that for a large enough volume, and for a long enough simulation run, the physical processes causing the density variations in the ISM in a galactic disc can be regarded as random and independent events. Therefore, the PDF of $\log$ (density) becomes Gaussian and the density PDF lognormal. The medium is inhomogeneous on a local scale, but in a quasi-steady state on a global scale.

Little observational evidence exists to test the results of the simulations. To date lognormal PDFs have been derived for the HI column densities in the LMC (Wada et al. 2000), for emission measures perpendicular to the Galactic plane of the DIG in the Milky Way (Hill et al. 2007, 2008), and for the local volume density of dust near stars within 400 pc of the Sun (Gaustad \& Van Buren, 1993). Here we present the PDFs of average volume densities of the DIG and the diffuse atomic gas in the Milky Way, and show that they are consistent with lognormal distributions as well.

We investigated the density PDFs of the DIG and of diffuse atomic hydrogen gas (HI) in the solar neighbourhood, using the dispersion measures of 34 pulsars at known distances (Berkhuijsen \& Müller, 2008) and HI absorption lines towards

\footnotetext{
${ }^{1}$ Max-Planck-Institut für Radioastronomie, Auf dem Hügel 69, 53121 Bonn, Germany.

2 School of Mathematics and Statistics, Newcastle University, NE1 7RU, U.K.
} 
2 The Role of Disk-Halo Interaction in Galaxy Evolution: Outflow vs Infall?
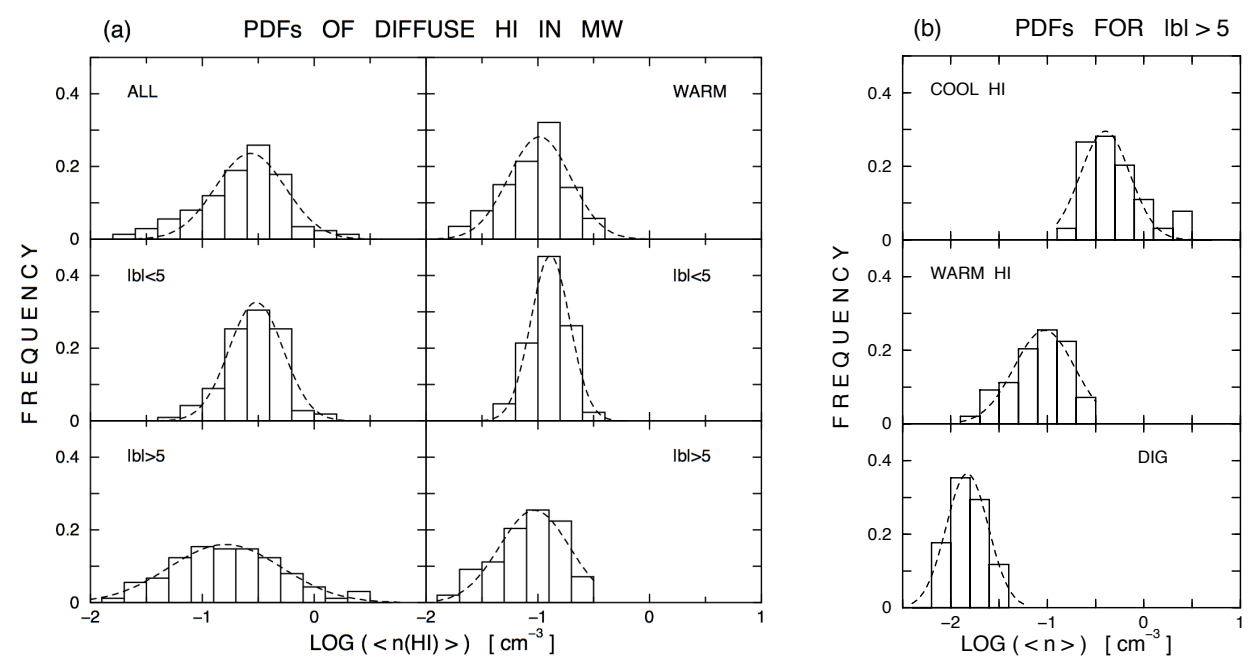

Fig. 1. (a) Six panels showing PDFs of HI. Left panels: PDFs of all diffuse HI, right panels: PDFs of only warm HI. (b) Three panels showing PDFs at high latitude ( $|b| \geq$ $5^{\circ}$ ) for two HI phases and the diffuse ionized gas.

375 stars (Diplas \& Savage, 1994a b). Further details and an extensive discussion of some of the results presented here are given in Berkhuijsen \& Fletcher (2008).

\section{Results and discussion}

In Fig. 1(a)(left panels) we present the PDFs of the average volume density of HI, $\left\langle n_{\mathrm{HI}}\right\rangle$, for the full sample of 375 stars (top) and for the stars at low $\left(|b|<5^{\circ}\right)$ and high $\left(|b| \geq 5^{\circ}\right)$ latitudes (middle and bottom panels) (Diplas \& Savage, 1994a). The PDF for all stars is approximately lognormal above $\log \left\langle n_{\mathrm{HI}}\right\rangle=-1$, but there is a clear excess at lower densities reflected in the large reduced- $\chi^{2}$ statistic (see Table 11. The PDFs for low and high $|b|$ have a lognormal shape but their maxima are shifted with respect to each other. The latter sample clearly causes the lowdensity excess in Fig. 1(a)(top left). This is also the case for the PDFs for the lines of sight (LOS) towards stars probing warm HI (Diplas \& Savage, 1994b) shown in Fig. 1(a) (right panels). The shifts between the maxima show the difference in mean density between low and high latitudes (see Table 1). Note that all densities of the warm sample have $\langle n\rangle<0.3 \mathrm{~cm}^{-3}$.

The dispersions of the PDFs of the high $|b|$ samples are twice those of the low $|b|$ samples. A possible explanation is that the low $|b|$ LOS cross more turbulent cells than at high $|b|$ (Vázquez-Semadeni \& Garcia, 2001).

Table 1 shows that the dispersions of the PDFs of the warm HI are slightly smaller than for the full sample. The combination of warm and cool (denser) gas in the full sample increases the dispersion because the density range becomes larger. 
Table 1. Lognormal fits to the PDFs of $\left\langle n_{\mathrm{HI}}\right\rangle$ and $\left\langle n_{\mathrm{e}}\right\rangle$. The fitted function is $Y=(\sqrt{2 \pi} \sigma)^{-1} \exp \left[-\left(\log _{10}\langle n\rangle-\mu\right)^{2} / 2 \sigma^{2}\right]$, where $Y$ is the normalised number of pulsars or stars per bin, and the reduced chi-square value for each fit is given.

\begin{tabular}{lrrrrr}
\hline & \multicolumn{4}{c}{ Position of maximum } & \multicolumn{2}{r}{ Dispersion } \\
Area & $N$ & $\mu$ & $\langle n\rangle$ & $\sigma$ & $\chi^{2}$ \\
\hline All stars & 375 & $-0.57 \pm 0.03$ & $0.27 \pm 0.02$ & $0.34 \pm 0.03$ & 6.0 \\
$|b|<5^{\circ}$ & 213 & $-0.52 \pm 0.02$ & $0.30 \pm 0.02$ & $0.24 \pm 0.02$ & 2.7 \\
$|b| \geq 5^{\circ}$ & 162 & $-0.80 \pm 0.02$ & $0.16 \pm 0.01$ & $0.50 \pm 0.02$ & 0.4 \\
& & & & & \\
Cool HI $|b| \geq 5^{\circ}$ & 64 & $-0.40 \pm 0.05$ & $0.40 \pm 0.04$ & $0.27 \pm 0.03$ & 1.3 \\
& & & & & \\
Warm HI & 140 & $-0.98 \pm 0.03$ & $0.10 \pm 0.01$ & $0.28 \pm 0.03$ & 2.5 \\
$|b|<5^{\circ}$ & 42 & $-0.89 \pm 0.01$ & $0.13 \pm 0.01$ & $0.18 \pm 0.01$ & 0.1 \\
$|b| \geq 5^{\circ}$ & 98 & $-1.07 \pm 0.03$ & $0.09 \pm 0.01$ & $0.33 \pm 0.03$ & 1.0 \\
& & & & & \\
DIG $|b| \geq 5^{\circ}$ & 34 & $-1.83 \pm 0.02$ & $0.015 \pm 0.001$ & $0.22 \pm 0.02$ & 0.3 \\
\hline
\end{tabular}

This is clearly visible in Fig. 1(b): addition of the samples at high $|b|$ of cool HI (top) and warm HI (middle) gives the distribution for all high $|b|$ LOS shown in the bottom-left panel of Fig. 1(a). However, the mixture of cool and warm HI only partly explains the difference in dispersion of the full samples at low and high $|b|$, because this difference also exists for the samples of warm HI.

In the bottom panel of Fig. 1(b) we show the PDF of DIG, which is also lognormal, below that of the warm HI at the same $|b|$. The temperatures of the two components are similar and if the ionized and atomic gas are well mixed, one would expect their dispersions to be the same. However, the dispersion of the DIG sample is about 30 per cent smaller than that of the warm HI sample (see Table1). A plausible explanation for the difference, which is also consistent with the higher density of the maximum in the diffuse $\left\langle n_{\mathrm{HI}}\right\rangle \mathrm{PDF}$, is that low density regions are more readily ionized than higher density gas and that the average degree of ionization of the diffuse gas is substantially lower than 50 per cent. We estimate the degree of ionization to be about 14 per cent, using the densities for $\left\langle n_{\mathrm{e}}\right\rangle$ and $\left\langle n_{\mathrm{HI}}\right\rangle$ in Table 1. Alternatively, the DIG could have a higher mean temperature than the warm $\mathrm{HI}$ but with a smaller temperature range; in the simulations of de Avillez \& Breitschwerdt (2005) high temperature gas indeed has a lower median density and smaller dispersion.

\section{Conclusions}

The results presented in Section 2 lead us to the following conclusions: 
4 The Role of Disk-Halo Interaction in Galaxy Evolution: Outflow vs Infall?

1. The density PDF of the diffuse ISM is approximately lognormal, but there is a significant low density excess in the best-fit distribution.

2. The density PDFs of the diffuse ISM in the disk $\left(|b|<5^{\circ}\right)$ and away from the disk $\left(|b| \geq 5^{\circ}\right)$ are lognormal, but the positions of their maxima and the dispersions differ. These differences produce the low density excess in the PDF for all lines of sight.

3. Several effects seem to influence the shape of the PDF. An increase of the number of clouds/cells along the LOS causes a decrease in the dispersion, whereas a mixture of cool (dense) and warm (less dense) gas causes an increase in the dispersion.

These results provide strong support for the existence of a lognormal density PDF in the diffuse (i.e. average densities of $n<1 \mathrm{~cm}^{-3}$ ) ionized and neutral components of the ISM. In turn, the form of the PDFs is consistent with the small-scale structure of the diffuse ISM being controlled by turbulence.

\section{References}

de Avillez, M. A. \& Breitschwerdt, D. 2005, A\&A, 436, 585

Berkhuijsen, E. M. \& Fletcher, A. 2008, MNRAS, 390, L19

Berkhuijsen, E. M. \& Müller, P. 2008, A\&A, 490, 179

Diplas, A. \& Savage, B. D. 1994a, ApJS, 93, 211

Diplas, A. \& Savage, B. D. 1994b, ApJ, 427, 274

Elmegreen, B. G. \& Scalo, J. 2004, ARA\&A, 42, 211

Gaustad, J. E. \& Van Buren, D. 1993, PASP, 105, 1127

Hill, A. S., Reynolds, R. J., Benjamin, R. A. \& Haffner, L. M. 2007, ASP Conf. Ser., 365,250

Hill, A. S., Benjamin, R. A., Kowal, G., Reynolds, R. J., Haffner, L. M. \& Lazarian, A. 2008, ApJ, 686, 363

Vázquez-Semadeni, E. \& Garcia, N. 2001, ApJ, 557, 727

Wada, K. \& Norman, C. A. 2007, ApJ, 660, 276

Wada, K., Spaans, M. \& Kim, S. 2000, ApJ, 540, 797 